\title{
Robótica Educacional como Estratégia de Promoção do Pensamento Computacional - Uma Proposta de Metodologia Baseada em Taxonomias de Aprendizagem
}

\author{
Christiano Avila, Simone Cavalheiro \\ ${ }^{1}$ Programa de Pós-Graduação em Computação - Universidade Federal de Pelotas (UFPel) \\ Pelotas - RS - Brazil \\ $\{\mathrm{cm}$.avila, simone.costa\}@inf.ufpel.edu.br
}

\begin{abstract}
This article presents a proposal for a methodology for gradual engagement of apprentices in concepts related to educational robotics. Robotics is used as a strategy for the dissemination of concepts related to Computational Thinking. The methodology also incorporates two learning taxonomies. The first, an adaptation of the revised Bloom taxonomy guides the level of cognitive requirement of the contents and activities that will be realized in future interventions based on the methodology. The other, known as SOLO taxonomy, is used to measure students' level of structural understanding regarding exercise I activity responses.
\end{abstract}

Resumo. Este artigo apresenta uma proposta de metodologia para engajamento gradativo de aprendizes em conceitos relacionados à robótica educacional. A robótica é utilizada como estratégia para disseminação de conceitos relacionados ao Pensamento Computacional. A metodologia também incorpora duas taxonomias de aprendizagem. A primeira, uma adaptação da taxonomia de Bloom revisada, orienta o nível de exigência cognitiva dos conteúdos $e$ atividades que serão realizadas em futuras intervenções baseadas na metodologia. A outra, conhecida por taxonomia SOLO, é utilizada para mensurar o nivel de entendimento estrutural dos alunos em relação às respostas de exercícios/atividades.

\section{Introdução}

A criação do termo "Pensamento Computacional" (PC) é comumente atribuído à pesquisadora Jeannette Wing [Barr et al. 2011, Lye and Koh 2014, Perković et al. 2010]. Certamente em função do artigo escrito por [Wing 2006] onde ela introduz PC como um processo que envolve a "resolução de problemas, a capacidade de projetar sistemas e a compreensão do comportamento humano recorrendo aos conceitos fundamentais da Ciência da Computação".

Para disseminar ou promover os conceitos relacionados ao PC são utilizadas várias estratégias. Uma delas é a robótica, uma ciência multidisciplinar por natureza que abrange a criação, construção e utilização de robôs. Envolve conceitos da mecânica, cinemática, automação, hidráulica, informática, inteligência artificial e outros.

Em [César 2014] são apresentadas algumas constatações sobre robótica educacional, ressaltando que a mesma: a) propicia a interação, cooperação e aprendizagem 
VI Congresso Brasileiro de Informática na Educação (CBIE 2017)

Anais dos Workshops do VI Congresso Brasileiro de Informática na Educação (WCBIE 2017)

coletiva; b) potencializa a ludicidade no relacionamento entre aprendiz e conhecimento; c) estimula a criatividade; d) desenvolve o raciocínio lógico; e) contribui para o desenvolvimento de aspectos ligados ao planejamento e organização de projetos; f) possibilita vivenciar e compreender o erro; g) permite a transformação do aprendiz de simples usuário a construtores de produtos tecnológicos, e h) estimula a exploração de conceitos ligados a pesquisa científica e a multi/trans/pluridisciplinaridade.

Assim, para que os alunos possam usufruir dos benefícios da robótica educacional, enquanto computação física (não simulada), é necessário utilizar algum tipo de dispositivo robótico. Algumas pesquisas [Benitti 2012, Neto et al. 2015, Bombasar et al. 2015, Ramos et al. 2015] indicam que os kits de robótica da empresa LEGO (LEGO Group) são os mais utilizados em projetos de robótica educacional.

Além dos dispositivos comerciais, existem diversas alternativas de equipamentos que estão sendo utilizados em robótica educacional e que que adotam a filosofia "open source" ou também nominado de "open hardware" ou ainda "open source hardware". São placas que podem ser copiadas/clonadas e utilizadas sem qualquer pagamento de licenças. Um dos exemplos mais conhecidos é a placa Arduino, lançada comercialmente em 2005 por Massimo Banzi [Severance 2014] e utilizada com frequência em projetos que envolvem robótica educacional [Baichtal et al. 2013, Lazar 2013, Monk 2013].

A utilização dos dispositivos citados anteriormente, de tecnologia proprietária ou "hardware livre", precisa ser realizada com aprendizes em projetos ancorados em conceitos pedagógicos bem definidos. Visando estruturar o nível de exigência cognitiva de intervenções que envolvem PC, ou mesmo para avaliar a efetividade, alguns estudos estão utilizado as taxonomias de aprendizagem [Falloon 2016, Mishra and Iyer 2015, Weiwei et al. 2015]. O termo taxonomia vem do grego taxis = ordenação e no$m o s=$ sistema, norma. Assim, as taxonomias de aprendizagem permitem classificar habilidades ou desempenho humano e consideram a natureza dos desempenhos, das habilidades ou dos processos ou condições em que ocorre a aprendizagem. As categorizações expressadas pelas taxonomia dos objetivos educacionais proposta por Benjamin Bloom [Bloom et al. 1956, Anderson et al. 2001] e de identificação de patamares de formalização do pensamento conhecida por SOLO e proposta por [Biggs and Collis 2014] são exemplos de taxonomias de aprendizagem. Tanto SOLO como Bloom são comumente encontradas na literatura como ferramentas para a elaboração de mecanismos de avaliação de aprendizagens e como balizadores para a elaboração de conteúdos programáticos (estrutura) de intervenções educacionais.

A taxonomia SOLO (Structure of Observing Learning Outcome), desenvolvida por Biggs e Collis [Biggs and Collis 2014], visa identificar o tipo de pensamento alcançado a partir das respostas de estudantes na realização de atividades/tarefas/exercícios. Ancorada em pressupostos Piagetianos, permite a elaboração de escalas de entendimento onde as respostas indicam os distintos níveis de complexidade no entendimento ou assimilação de conteúdos.

Para Biggs e Collis o desempenho não está vinculado diretamente às capacidades cognitivas de certa fase da vida. Eles entendem que uma mesma pessoa pode estar em algumas fases de forma simultânea, dependendo da área do conhecimento, ou seja, o desempenho está diretamente relacionado com a área do conhecimento. Na taxonomia 
VI Congresso Brasileiro de Informática na Educação (CBIE 2017)

Anais dos Workshops do VI Congresso Brasileiro de Informática na Educação (WCBIE 2017)

SOLO existem cinco níveis de complexidade do modo estrutural de funcionamento do pensamento. São eles: a) pré-estrutural onde as respostas são totalmente inadequadas; b) uniestrutural onde é possível identificar um (ou poucos) aspecto(s) relevantes; c) multiestrutural onde se consegue identificar alguns aspectos relevantes; d) relacional onde os aspectos relevantes são relacionados; e e) abstrato estendido onde o aluno apresenta a capacidade de generalizar a estrutura para um novo quadro com características mais abstratas.

No que diz respeito à utilização da taxonomia SOLO em projetos relacionados ao PC, em [Seiter 2015] foram propostos desafios a serem resolvidos por meio de programação com Scratch. A avaliação foi realizada de acordo com um "gabarito" que facilita o enquadramento do código em um determinado nível da taxonomia SOLO, ou seja, o nível de entendimento alcançado pelo aluno em relação a estrutura do problema. Outro projeto, uma adaptação da taxonomia, foi utilizada em [Biró et al. 2015] para avaliar a realização de duas atividades por alunos iniciantes em um curso superior de informática. A avaliação das tarefas exigiu uma análise detalhada para descobrir como os alunos resolveram as tarefas e como suas habilidades algorítmicas estão desenvolvidas.

Por outro lado, a taxonomia dos objetivos educacionais (taxonomia de Bloom), estrutura-se como framework que permite classificar assuntos que se espera ou se pretende que os alunos aprendam como resultado de algum processo educativo [Krathwohl 2002]. De forma mais abrangente o grupo de autores que criou a taxonomia verificou que seria desejável, dentre outras características [Ferraz et al. 2010]: a) padronizar a linguagem sobre os objetivos de aprendizagem facilitando a comunicação entre os educadores; b) servir como base para a definição dos objetivos de currículos de cursos visando a definição mais clara de um perfil discente; e c) sincronizar os objetivos educacionais das intervenções (curso, aula, oficina) com a avaliação;

A taxonomia, que na versão original acabou sendo lançada em 1956, oficialmente foi nomeada de "Taxonomia dos objetivos educacionais" mas acabou ficando conhecida, em função da liderança do pedagogo e psicólogo norte-americano Benjamin Bloom ${ }^{1}$, por "Taxonomia de Bloom". Considerando a importância da taxonomia desenvolvida em 1956 e os avanços psico-pedagógicos que ocorreram desde o lançamento da versão original, uma versão revisada da taxonomia foi lançada em $2001 \mathrm{em}$ um livro chamado "A taxonomy for learning, teaching and assessing: a revision of Bloom's taxonomy for educational objectives" [Anderson et al. 2001].

Uma das alterações realizadas foi a transformação em uma taxonomia bidimensional, com a criação de uma dimensão para separar o conhecimento, um substantivo que seria "o que" (na vertical) e o verbo em uma dimensão dos processo cognitivos relacionada ao "como" (na horizontal). Os níveis, representados por verbos, iniciam por 1-lembrar (mais concreto e simples), passando por 2-entender, 3-aplicar, 4-analisar, 5avaliar, chegando ao 6-criar (mais abstrato e complexo).

A utilização de taxonomias de aprendizagem na área de computação (em geral) já

\footnotetext{
${ }^{1}$ Benjamin Bloom - "Pedagogo e psicólogo norte-americano, nascido em 1913 e falecido em 1999, lecionou na Universidade de Chicago onde desenvolveu investigações sobre os processos de planificação $e$ avaliação no ensino. Teve uma influência decisiva na área das ciências da educação ao propor uma taxionomia dos objetivos educacionais” [Infopedia 2017].
} 
VI Congresso Brasileiro de Informática na Educação (CBIE 2017)

Anais dos Workshops do VI Congresso Brasileiro de Informática na Educação (WCBIE 2017)

é uma realidade, pois diversos estudos foram publicados com foco em área distintas como, por exemplo, em programação de computadores onde em [Scaico et al. 2012] os autores apresentam uma proposta de ensino introdutório de programação (Python) por meio de um jogo que "oferece várias possibilidades de desafios, que estão organizados em função da taxonomia de Bloom" [Scaico et al. 2012]. Também com foco na introdução de conceitos de programação, em [de Jesus and Raabe 2009] é discutido como cada uma das categorias da taxonomia vêm sendo utilizada em avaliações de programação. Também apresenta um instrumento de avaliação onde as questões são classificada de acordo com a taxonomia de Bloom. Em [Whalley et al. 2006] o estudo, fundamentado nas taxonomias de Bloom e SOLO, apresenta um instrumento que visa avaliar a capacidade de alunos, iniciantes em programação, em relação à leitura e entendimento dos códigos desenvolvidos.

A taxonomia de Bloom também foi utilizada como fundamento para a construção de uma ferramenta de auto-avaliação, um questionário de pesquisa que usa a taxonomia revisada da Bloom como base de sua escala [Alaoutinen and Smolander 2010]. Também tendo a taxonomia de Bloom como fundamento, um teste nacional aplicado anualmente pelo ministério da educação de Israel procura identificar a proficiência dos alunos (7aㅡ, $8^{\text {a }}$ e 9a séries) em relação ao ambiente de programação Scratch [Zur-Bargury et al. 2013].

Sendo assim, este trabalho apresenta uma metodologia para intervenções, baseada em taxonomias de aprendizagem e que envolvam a promoção do PC por meio da robótica educacional. A partir da metodologia proposta será possível estruturar intervenções de robótica educacional para jovens do ensino básico, em especial o fundamental, onde o envolvimento com a robótica e a complexidade ou exigência cognitiva será aumentada de forma gradativa. O texto está organizado da seguinte forma. A Seção 2 descreve a metodologia que está sendo proposta (estruturada em cinco fases). Na seção 3 são relacionadas algumas conclusões e uma proposta de trabalhos futuros que trata de uma instância ou efetivação prática da proposta apresentada. Por fim são apresentadas as referências bibliográficas.

\section{Proposta de Metodologia para Intervenções com Robótica}

A questão metodológica é um dos aspectos mais importantes no planejamento de uma intervenção com robótica educacional. É importante definir a concepção pedagógica e, a partir desta macro-definição, planejar como serão as intervenções. Seguindo uma linha construcionista, a intervenção não deve ocorrer como aulas tradicionais/instrucionais, onde um professor ensina e os alunos, mecanicamente, "aprendem" aquilo que o professor ensinou.

Em [Rusk et al. 2008] os autores citam algumas estratégias a serem levadas em consideração. A primeira é "focar em temas e não apenas em desafios específicos" fornecendo mais possibilidades para que os aprendizes pensem em novos problemas e não apenas na solução de um problema específico. A segunda estratégia recomenda trabalhar de forma interdisciplinar, "combinar arte e engenharia" como forma de maior engajamento dos participantes. A terceira é "incentivar a narração de histórias" para atender ao perfil/estilo de aluno (dramaturgo versus padronizadores). Por fim "organizar exibições" como uma oportunidade para os jovens apresentarem os seus trabalhos numa exposição, eventualmente para a família e membros da comunidade.

A proposta de metodologia apresentada nesta seção é uma adaptação baseada, 
fundamentalmente, na combinação de dois trabalhos. O primeiro, [Komis et al. 2017] trata de uma taxonomia de aprendizagem voltada à intervenções de robótica educacional onde o envolvimento dos alunos com os dispositivos robóticos é feito de forma gradual e em cinco fases que são: 1) exposição passiva à robótica (sem manipulação); 2) discussão sobre robótica (Sem Programação); 3) robótica processual individual ou colaborativa (programação / sem construção); 4) robótica orientada a engenharia (programação e construção); e 5) Desafio robótico co-criativo orientado a projetos (Definição, Programação e Construção Colaborativa de Projetos). O segundo trabalho [Fuller et al. 2007], que combinado com o primeiro, formam a base desta proposta, é uma adaptação da taxonomia de Bloom para a ciência da computação. A proposta está baseada em uma matriz bidimensional, apresentada na Figura 1, onde os níveis da taxonomia (dimensão dos processos cognitivos) são distribuídos, uma parte na vertical (criar e aplicar) e outra parte na horizontal (lembrar, entender, analisar e avaliar).

A matriz será utilizada nesta proposta de metodologia dentro das cinco fases de [Komis et al. 2017], para orientar o nível de exigência cognitiva das atividades relacionadas ao desenvolvimento de conceitos e habilidades do PC por meio de atividades relacionadas à programação e robótica.

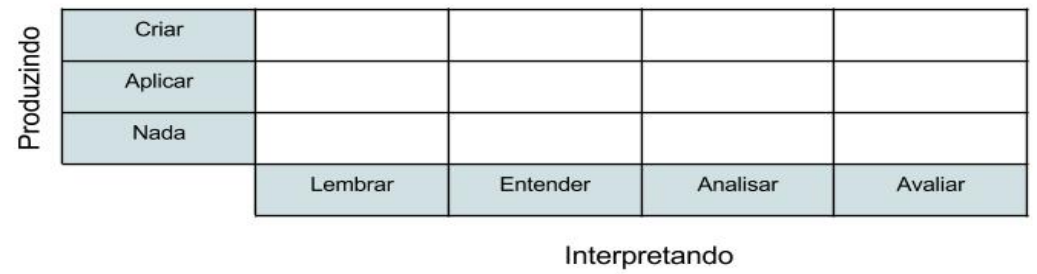

\section{Figura 1. Uma apresentação gráfica da adaptação bidimensional da taxonomia da Bloom - Fonte: [Fuller et al. 2007]}

A seguir serão descritas as as cinco fases que compõe a metodologia que objetiva, de forma gradativa, desenvolver conceitos e habilidades relacionadas ao PC tendo a robótica educacional como estratégia.

\subsection{Fase 1 - Exposição passiva à robótica (sem manipulação)}

Nesta fase, de atividades preparatórias, os professores devem abordar o tema PC, programação de computadores e robótica. Os recursos didáticos a serem utilizados serão slides, vídeos e demonstrações onde os alunos observam um robô e suas diferentes capacidades. O conceito/formato da programação, realizada em blocos, também deve ser apresentada aos alunos. As atividades a serem desenvolvidas, de acordo com a tabela bidimensional (Figura 1) envolvem os níveis "Nada - Lembrar" e "Nada - Entender" onde as atividades/exercícios irão abordar os conceitos para verificar se um vocabulário e conhecimentos básicos foram memorizados e entendidos.

Em relação a avaliação, nesta primeira fase, é adequado mensurar o nível de entendimento estrutural alcançado pelos alunos utilizando a taxonomia SOLO [Biggs and Collis 2014]. Devem ser desenvolvidas questões discursivas, a serem respondidas individualmente, onde as respostas serão classificadas de acordo com a taxonomia SOLO. Carga horária estimada: $6 \mathrm{~h}$ distribuídas em 3 encontros de $2 \mathrm{~h}$. 
VI Congresso Brasileiro de Informática na Educação (CBIE 2017)

Anais dos Workshops do VI Congresso Brasileiro de Informática na Educação (WCBIE 2017)

\subsection{Fase 2 - discussão sobre robótica (Sem Programação)}

Nesta fase, que ainda é preparatória, os participantes devem ser estimulados a exporem suas opiniões e conhecimento a respeito da robótica. Devem falar sobre os componentes, comportamento e questões relacionadas ao seu uso na sociedade. A discussão deve permitir a construção, em conjunto, de novos significados e pode ser usada para desenvolver o pensamento crítico em torno da robótica na educação e as repercussões desta tecnologia em outras áreas. As discussões devem levar os alunos ao nível "Nada - Entender" da tabela bidimensional onde os alunos deverão entender não só as questões técnicas relacionadas à robótica, mas também os diversos efeitos na sociedade.

A avaliação também deve ser realizada por meio do entendimento estrutural da taxonomia SOLO, porém mais focado em um entendimento mais amplo das discussões realizadas nesta etapa. Carga horária estimada: $2 \mathrm{~h}$ distribuídas em 1 encontro de $2 \mathrm{~h}$.

\subsection{Fase 3 - robótica processual individual ou colaborativa (programação / sem construção)}

Nesta fase deve ocorrer uma imersão na programação que será utilizada, posteriormente, para controlar os dispositivos robóticos. Os alunos deverão adquirir fluência nos comandos e estrutura da linguagem, porém, nesta etapa, ainda sem manipular ou executar a programação nos dispositivos físicos. Pode-se utilizar a estratégia de roteiros (passo a passo) onde os alunos, individualmente ou em duplas, desenvolvem programas e realizam desafios para exercitar os conteúdos adquiridos durante a execução dos roteiros. Os desafios devem ser do tipo "responda ou explique o que faz um determinado programa", "desenvolva os códigos que estão faltando", "altere o código para atender a tal comportamento" e "faça um programa com as seguintes características".

Serão exercitadas, nesta fase, as competências básicas de programação (pensamento algorítmico e resolução de problemas). Assim, a realização de roteiros e desafios deve ocorrer em dois momentos. Um primeiro onde os alunos irão trabalhar apenas com programação, sem utilizar os dispositivos robóticos e, em um segundo momento, os roteiros contemplarão a execução de programação para controlar os dispositivos, porém sem montagem, apenas a execução em dispositivos previamente montados pelos professores. A estratégia de trabalhar, em um primeiro momento, exclusivamente com programação, sem a manipulação dos dispositivos robóticos, visa evitar a sobrecarga cognitiva dos participantes.

A execução dos roteiros e a realização dos desafios deve exigir dos alunos um esforço cognitivo em diversos níveis da tabela bidimensional que são: a) "Nada - Entender" na execução dos roteiros; b) "Nada - Entender" para responder sobre o funcionamento de um código existente; c) "Aplicar - Entender" para preencher códigos que faltam em um programa e para alterar código para atender a um determinado comportamento; d) "Criar - Analisar" para desenvolver novos códigos.

Para avaliar esta fase recomenda-se a utilização do modelo proposto em [Seiter 2015] onde o código desenvolvido pelos alunos, para responder aos desafios, é avaliado segundo um "gabarito" que permite classificar a produção dos alunos pela taxonomia SOLO. O gabarito pode ajudar a identificar o nível de entendimento dos alunos em conceitos mais específicos da programação como seleção/condição, repetição/laços, variáveis e paralelismo. Para atividades desenvolvidas com o ambiente Scratch é possível 
VI Congresso Brasileiro de Informática na Educação (CBIE 2017)

Anais dos Workshops do VI Congresso Brasileiro de Informática na Educação (WCBIE 2017)

utilizar ferramentas automatizadas para análise do código produzido como, por exemplo, o software Dr. Scratch [Moreno-León and Robles 2015b, Moreno-León et al. 2015, Moreno-León and Robles 2015a] que atribui um escore para a competência do desenvolvedor em sete conceitos: 1) abstração e decomposição de problemas; b) pensamento lógico; 3) sincronização; 4) paralelismo; 5) noções algorítmicas de controle de fluxo; 6) interatividade com o usuário; e 7) representação de dados. Carga horária estimada: 10h distribuídas em 5 encontro de $2 \mathrm{~h}$.

\subsection{Fase 4 - robótica orientada a engenharia (programação e construção)}

A fase 4 é similar à anterior, porém difere no quesito construção/montagem dos dispositivos que, nesta etapa, é exigida dos participantes. Assim, os dispositivos devem ser entregues sempre desmontados aos alunos. A fase 4 terá duas subfases, uma primeira onde os participantes seguirão roteiros para orientar na montagem e, em uma segunda onde será solicitado que os participantes atendam aos desafios sem ter um roteiro para a montagem dos dispositivos. Recomenda-se a formação de grupos (3 ou 4 componentes) para a execução dos roteiros e atividades.

As características relacionadas a matriz bidimensional, desenvolvimento de conceitos e habilidades do PC e de avaliação são idênticas à fase anterior, porém acrescida do nível "Aplicar - Analisar", principalmente pela questão da montagem dos dispositivos, onde é necessário entender a inter-relação existente entre as partes dos componentes. Carga horária estimada: $8 \mathrm{~h}$ distribuídas em 4 encontro de $2 \mathrm{~h}$.

\subsection{Fase 5 - Desafio robótico co-criativo orientado a projetos (Definição, Programação e Construção Colaborativa de Projetos)}

Nesta etapa, de desafios co-criativos em robótica, os alunos são encorajados a resolver problemas reais que serão escolhidos de forma participativa. Para resolver os problemas deve-se utilizar a estratégias de aprendizagem baseada em projetos que estruturam e ajudam a desenvolver soluções criativas para problemas reais (por exemplo, projetar e programar um conjunto de dispositivos robóticos para irrigar automaticamente uma horta).

Uma questão a ser organizada no planejamento desta fase é a maior ou menor disponibilidade de equipamentos (ou de recursos para aquisição) que possam dar suporte aos projetos. Quando essa disponibilidade é limitada, é necessário elaborar um "cardápio" ou "portfólio" de "projetos possíveis" de serem realizados. Isso, obviamente, limita ou direciona a criatividade dos participantes. Em relação a esses dispositivos, recomenda-se que sejam disponibilizadas placas controladoras do tipo arduino ou compatíveis, atuadores e sensores que permitam a execução de diversos tipos de projetos. Também é recomendável a disponibilidade de equipamentos do tipo impressora 3D, fresadora $\mathrm{CNC}$ em um espaço comumente chamado de FabLab ou espaço maker.

Nesta fase, serão explorados os níveis "Aplicar - Entender" e "Criar - Entender" da tabela bidimensional. Isso exige um considerável esforço cognitivo pois é necessário que os alunos organizem uma determinada ideia, realizem o planejamento detalhado e a posterior execução (produção). Segundo [Faria et al. 2010] não é simples determinar se a tarefa pertence ao nível aplicação ou criação. Além disso, o tamanho do problema tem influência na dificuldade para a execução, porém também não determina se a tarefa se enquadra em aplicação ou criação. Portanto o nível cognitivo atingido na execução deve variar em função do ineditismo e criatividade aplicados nos projetos. 
VI Congresso Brasileiro de Informática na Educação (CBIE 2017)

Anais dos Workshops do VI Congresso Brasileiro de Informática na Educação (WCBIE 2017)

\section{Conclusões}

Este artigo apresentou uma proposta de metodologia para orientar a construção de intervenções que visem desenvolver conceitos e habilidades relacionadas ao PC. A proposta sugere a execução em cinco fases de gradativo engajamento em atividades de robótica educacional.

Em relação aos níveis cognitivos da taxonomia de Bloom, a presente proposta de metodologia foi construída tendo como base a adaptação descrita em [Fuller et al. 2007] e, considerando uma intervenção de aproximadamente 34 horas, chegou a seguinte distribuição: a) "Nada - Lembrar" com 3h (9 \%); b) "Nada - Entender" com 12,5h (37\%); c) "Aplicar - Entender" com 9,5h (28\%); d) "Aplicar - Analisar" com 3h (9\%); f) "Criar - Entender" com 2h (6\%); g) "Criar - Analisar" com 2h (6\%); e h) "Criar - Avaliar" com $2 \mathrm{~h}(6 \%)$.

Em relação a trabalhos futuros, já está em elaboração uma instância prática desta metodologia, com uma proposta de conteúdos onde serão especificados os aplicativos (Scratch, S4A, Enchanting e outros), dispositivos robóticos (Arduino, Lego), atividades e desafios com a definição específica dos respectivos níveis cognitivos e conceitos, habilidades e competências do PC a serem desenvolvidos. Assim, será realizado um estudo de caso, por meio da realização de uma intervenção com alunos do ensino fundamental, o qual permitirá a coleta e análise de dados visando avaliar a efetividade da metodologia proposta.

\section{Referências}

Alaoutinen, S. and Smolander, K. (2010). Student self-assessment in a programming course using bloom's revised taxonomy. In Proceedings of the 15 o annual conference on Innovation and technology in computer science education, pages 155-159. ACM.

Anderson, L. W., Krathwohl, D. R., Airasian, P., Cruikshank, K., Mayer, R., Pintrich, P., Raths, J., and Wittrock, M. (2001). A taxonomy for learning, teaching and assessing: A revision of bloom's taxonomy. New York. Longman Publishing. Artz, AF, \& ArmourThomas, E.(1992). Development of a cognitive-metacognitive framework for protocol analysis of mathematical problem solving in small groups. Cognition and Instruction, 9(2):137-175.

Baichtal, J., Beckler, M., and Wolf, A. (2013). Chapter 1. project: Drawbot. https: //goo.gl/bMGGXu. Acesso em: 2017-20-02.

Barr, D., Harrison, J., and Conery, L. (2011). Computational thinking: A digital age skill for everyone. Learning \& Leading with Technology, 38(6):20-23.

Benitti, F. B. V. (2012). Exploring the educational potential of robotics in schools: A systematic review. Computers \& Education, 58(3):978-988.

Biggs, J. B. and Collis, K. F. (2014). Evaluating the quality of learning: The SOLO taxonomy (Structure of the Observed Learning Outcome). Academic Press.

Biró, P., Csernoch, M., Máth, J., and Abari, K. (2015). Measuring the level of algorithmic skills at the end of secondary education in hungary. Procedia - Social and Behavioral Sciences, 176:876 - 883. IETC 2014, 3-5 September 2014, Chicago, IL, USA. 
VI Congresso Brasileiro de Informática na Educação (CBIE 2017)

Anais dos Workshops do VI Congresso Brasileiro de Informática na Educação (WCBIE 2017)

Bloom, B. S., Engelhart, M. D., Furst, E. J., Hill, W. H., and Krathwohl, D. R. (1956). Taxonomy of educational objectives, handbook I: The cognitive domain, volume 19. New York: David McKay Co Inc.

Bombasar, J., Raabe, A., Miranda, E. M. d., and Santiago, R. (2015). Ferramentas para o ensino-aprendizagem do pensamento computacional: onde está alan turing? In Anais do SBIE.

César, D. R. (2014). Robótica pedagógica livre: uma alternativa metodológica para a emancipação sociodigital e a democratização do conhecimento.

de Jesus, E. A. and Raabe, A. L. A. (2009). Interpretações da taxonomia de bloom no contexto da programaçao introdutória. In Brazilian Symposium on Computers in Education (Simpósio Brasileiro de Informática na Educação-SBIE), volume 1.

Falloon, G. (2016). An analysis of young students' thinking when completing basic coding tasks using scratch jnr. on the ipad. Journal of Computer Assisted Learning, 32(6):576 - 593 .

Faria, E. S. J. d. et al. (2010). Método trifásico de ensino-aprendizagem baseado na taxionomia de objetivos educacionais de bloom: uma aplicação no ensino de programação de computadores.

Ferraz, A., Belhot, R. V., et al. (2010). Taxonomia de bloom: revisão teórica e apresentação das adequações do instrumento para definição de objetivos instrucionais. Gest. Prod., São Carlos, 17(2):421-431.

Fuller, U., Johnson, C. G., Ahoniemi, T., Cukierman, D., Hernán-Losada, I., Jackova, J., Lahtinen, E., Lewis, T. L., Thompson, D. M., Riedesel, C., and Thompson, E. (2007). Developing a computer science-specific learning taxonomy. In Working Group Reports on ITiCSE on Innovation and Technology in Computer Science Education, ITiCSEWGR '07, pages 152-170, New York, NY, USA. ACM.

Infopedia (2017). Benjamin bloom - infopedia. https://www.infopedia.pt/ \$benjamin-bloom.

Komis, V., Romero, M., and Misirli, A. (2017). A scenario-based approach for designing educational robotics activities for co-creative problem solving. Advances in Intelligent Systems and Computing, 560:158-169. cited By 0.

Krathwohl, D. R. (2002). A revision of bloom s taxonomy: An overview. Theory into practice, 41(4):212-218.

Lazar, J. (2013). Arduino and LEGO projects. Apress.

Lye, S. Y. and Koh, J. H. L. (2014). Review on teaching and learning of computational thinking through programming: What is next for k-12? Computers in Human Behavior, 41:51-61.

Mishra, S. and Iyer, S. (2015). An exploration of problem posing-based activities as an assessment tool and as an instructional strategy. Research and Practice in Technology Enhanced Learning, 10(1):5.

Monk, S. (2013). Programação com Arduino: Começando com Sketches-Série Tekne. AMGH Editora. 
VI Congresso Brasileiro de Informática na Educação (CBIE 2017)

Anais dos Workshops do VI Congresso Brasileiro de Informática na Educação (WCBIE 2017)

Moreno-León, J. and Robles, G. (2015a). Analyze your scratch projects with dr. scratch and assess your computational thinking skills. In Scratch Conference, pages 12-15.

Moreno-León, J. and Robles, G. (2015b). Dr. scratch: A web tool to automatically evaluate scratch projects. In Proceedings of the workshop in primary and secondary computing education, pages 132-133. ACM.

Moreno-León, J., Robles, G., and Román-González, M. (2015). Dr. scratch: Automatic analysis of scratch projects to assess and foster computational thinking. RED. Revista de Educación a Distancia, (46):1-23.

Neto, R. P. B., Santana, A. M., Rocha, D. P., and Souza, A. (2015). Robótica na educação: Uma revisão sistemática dos últimos 10 anos. In Brazilian Symposium on Computers in Education (SBIE), page 386.

Perković, L., Settle, A., Hwang, S., and Jones, J. (2010). A framework for computational thinking across the curriculum. In Proceedings of the fifteenth annual conference on Innovation and technology in computer science education, pages 123-127. ACM.

Ramos, E. et al. (2015). Pensamento computacional na escola e práticas de avaliação das aprendizagens. uma revisão sistemática da literatura. Challenges 2015: Meio Século de TIC na Educação, Half a Century of ICT in Education.

Rusk, N., Resnick, M., Berg, R., and Pezalla-Granlund, M. (2008). New pathways into robotics: Strategies for broadening participation. Journal of Science Education and Technology, 17(1):59-69.

Scaico, P., Marques, D. L., Melo, L. d. A., Azevedo, M. A., Neto, S. V. M., Oliveira, A., Júnior, J. A., Labanca, M., and Scaico, A. (2012). Um jogo para o ensino de programação em python baseado na taxonomia de bloom. In Anais do Congresso da Sociedade Brasileira de Computação-XX WEI.

Seiter, L. (2015). Using solo to classify the programming responses of primary grade students. In Proceedings of the 46th ACM Technical Symposium on Computer Science Education, SIGCSE '15, pages 540-545, New York, NY, USA. ACM.

Severance, C. (2014). Massimo banzi: Building arduino. Computer, 47(1):11-12.

Weiwei, C., Zhigang, L., Aihua, B., and Yanqin, T. (2015). Curriculum design for computational thinking training based on concept map. In 2015 7th International Conference on Information Technology in Medicine and Education (ITME), pages 413-416.

Whalley, J. L., Lister, R., Thompson, E., Clear, T., Robbins, P., Kumar, P., and Prasad, C. (2006). An australasian study of reading and comprehension skills in novice programmers, using the bloom and solo taxonomies. In Proceedings of the 8th Australasian Conference on Computing Education-Volume 52, pages 243-252. Australian Computer Society, Inc.

Wing, J. M. (2006). Computational thinking. Communications of the ACM, 49(3):33-35.

Zur-Bargury, I., Pârv, B., and Lanzberg, D. (2013). A nationwide exam as a tool for improving a new curriculum. In Proceedings of the 18th ACM Conference on Innovation and Technology in Computer Science Education, ITiCSE '13, pages 267-272, New York, NY, USA. ACM. 